\title{
Arachidonic Acid from Fungi Utilizing Fatty Acids with Shorter Chains as Sole Sources of Carbon and Energy
}

\author{
By S. S. RADWAN*† AND ARWA H. SOLIMAN \\ Department of Botany, Faculty of Science, Ain Shams University, Abbassia, Cairo, Egypt
}

(Received 2 June 1987; revised 11 September 1987)

\begin{abstract}
Several species of soil fungi, belonging to the genera Aspergillus, Penicillium, Fusarium, Paecilomyces, Trichoderma, Cladosporium and Stachybotrys could utilize caprylic, myristic, palmitic, stearic and oleic acids, as well as their sodium salts, as sole sources of carbon and energy. None of the fungal isolates showed any particular specificity to a given fatty acid. Only the caprylic acid utilizers grew poorly, otherwise the growth was fair to abundant. The optimal fatty acid concentration for fungal growth was $10 \mathrm{~g}^{-1}$. Growth yields were comparable with those on glucose. The optimal $\mathrm{pH}$ values were in the region of neutrality and the isolates could tolerate high acidity. Lipids from fungi grown on fatty acids or their sodium salts contained free fatty acids as the predominant lipid class. Such lipids were much richer in arachidonic acid than those from the same fungi grown on glucose. Fungi that contained the highest levels of arachidonic acid were those grown on myristic and palmitic acids, namely Aspergillus versicolor and Aspergillus ustus, respectively.
\end{abstract}

\section{INTRODUCTION}

There are very few studies on the microbial degradation of individual fatty acids in sewage (for a relevant review see Harp, 1979). Moreover, there are no systematic studies on microorganisms that can utilize fatty acids as sole sources of carbon and energy. The objectives of the present paper were to provide this information and to investigate the influence of fatty acids on the lipid composition of the micro-organisms utilizing them. Particular emphasis has been placed on the production of arachidonic acid $(20: 4)$ by fatty acid utilizing organisms.

\section{METHODS}

Fatty acids. Caprylic (8:0), myristic (14:0), palmitic (16:0), stearic (18:0) and oleic $(18: 1)$ acids were purchased from Merck. Sodium salts of these fatty acids were prepared by saponification with $\mathrm{NaOH}$ following established procedures (Christie, 1973).

Biodegradation of fatty acids. Suspensions of a garden soil sample and a desert soil sample were prepared by shaking $1 \mathrm{~kg}$ of each sample in 11 sterile water for $15 \mathrm{~min}$. After standing for $1 \mathrm{~h}$, the supernatant was decanted and $100 \mathrm{ml}$ samples were dispensed into $500 \mathrm{ml}$ Erlenmeyer flasks. For control experiments, flasks containing soil suspensions were sterilized by autoclaving at $120^{\circ} \mathrm{C}$ for $20 \mathrm{~min}$. Each flask was provided with $100 \mathrm{mg}$ of a fatty acid or its sodium salt and incubated at $30^{\circ} \mathrm{C}$. Flasks were assayed after 6,12 and 24 weeks to detect any undegraded fatty acids. Suspensions that had received sodium salts of fatty acids were first acidified with $6 \mathrm{M}-\mathrm{HCl}$ to release the fatty acids. Undegraded fatty acids were recovered by extracting three successive times with diethyl ether. The recovered fatty acids were dried, weighed and tested for purity by TLC.

Isolation of fatty acid utilizers. Micro-organisms capable of utilizing fatty acids as sole sources of carbon and energy were isolated on a basal medium supplemented with $250 \mathrm{mg} \mathrm{l}^{-1}$ of one fatty acid (dissolved in diethyl ether, which was evaporated leaving the acid dispersed in the medium) or its sodium salt. The fatty acids remained insoluble in the medium whereas the sodium salts were soluble, but only at neutral to alkaline $\mathrm{pH}$ values. The basal medium contained $\left(1^{-1}\right): 1.0 \mathrm{~g} \mathrm{KH}_{2} \mathrm{PO}_{4} ; 3.0 \mathrm{~g} \mathrm{NaCl} ; 0.25 \mathrm{~g} \mathrm{MgSO}_{4} .6 \mathrm{H}_{2} \mathrm{O} ; 1.0 \mathrm{~g} \mathrm{NaHCO} ; 0.5 \mathrm{~g}\left(\mathrm{NH}_{4}\right)_{2} \mathrm{SO}_{4}$; $1.0 \mathrm{~g} \mathrm{KNO}_{3} ; 0.25 \mathrm{~g} \mathrm{CaCl}_{2} . \mathrm{H}_{2} \mathrm{O} ; 0.1 \mathrm{mg} \mathrm{FeSO} \mathrm{F}_{4} .7 \mathrm{H}_{2} \mathrm{O} ; 0.1 \mathrm{mg} \mathrm{CuSO}{ }_{4} .5 \mathrm{H}_{2} \mathrm{O} ; 0.1 \mathrm{mg} \mathrm{ZnCl} .6 \mathrm{H}_{2} \mathrm{O} ; 0.5 \mathrm{mg}$

† Present address: Chairman, Department of Botany and Microbiology, Faculty of Science, Kuwait University, PO Box 5969, 13060 Safat, Kuwait. 
$\mathrm{MnCl}_{2} .4 \mathrm{H}_{2} \mathrm{O} ; 0.01 \mathrm{mg} \mathrm{MoO}_{3} ; 5.0 \mathrm{mg} \mathrm{H} \mathrm{H}_{3} \mathrm{BO}_{4} ; 0.01 \mathrm{mg} \mathrm{Al}\left(\mathrm{SO}_{4}\right)_{3} .14 \mathrm{H}_{2} \mathrm{O} ; 100 \mathrm{mg} \mathrm{CoSO} . \mathrm{H}_{2} \mathrm{O} ; 100 \mathrm{mg}$ $\mathrm{NiCl}_{2} .6 \mathrm{H}_{2} \mathrm{O} ; 25 \mathrm{mg} \mathrm{KI} ; 500 \mu \mathrm{g}$ thiamin; $500 \mu \mathrm{g}$ calcium pantothenate; $100 \mu \mathrm{g}$ folic acid; $500 \mu \mathrm{g}$ pyridoxin; $500 \mu \mathrm{g} p$-aminobenzoic acid; $500 \mu \mathrm{g}$ nicotinic acid; $50 \mu \mathrm{g}$ inositol; $20 \mu \mathrm{g}$ vitamin $\mathrm{B}_{12} ; 1.0 \mu \mathrm{g}$ biotin; and $20 \mathrm{~g}$ agar. The pH was adjusted to either 7.0 or 5.5 to favour bacteria and fungi, respectively. Incubation was at $30^{\circ} \mathrm{C}$. It was confirmed that each isolate could not grow on the basal medium in the absence of the respective fatty acid or its salt. Fungal isolates were identified by consulting Booth (1971), Raper \& Fennell (1977), Pitt (1979) and Domasch et al. (1980).

Extraction and analysis of lipids. Micro-organisms were grown in the basal medium containing the individual fatty acids or their sodium salts $\left(1 \mathrm{~g}^{-1}\right)$ under continuous shaking at $30^{\circ} \mathrm{C}$. Mycelia were harvested by filtration after $10 \mathrm{~d}$ and washed three successive times with boiling water to remove attached material and inactivated lipases. Total lipids were extracted three successive times from the mycelia with chloroform/methanol $(2: 1, \mathrm{v} / \mathrm{v})$ and purified as described by Folch et al. (1957). As a rule, boiling solvents were used in the first extraction only in order to inactivate any residual lipases (Kates, 1972). As far as feasible, all steps of washing and extraction were done in an atmosphere of oxygen-free nitrogen. The lipid composition was studied by TLC on layers of silica gel. Nonpolar classes were resolved as described by Mangold \& Malins (1960) using the developing solvent hexane/diethyl ether/acetic acid $(90: 10: 1$, by vol.). Polar classes were resolved by two-dimensional TLC using the solvent chloroform/methanol/7 M-ammonium hydroxide $(65: 30: 4$, by vol.) in the first direction and chloroform/methanol/acetic acid/water $(170: 25: 25: 4$, by vol.) in the second direction (Nichols, 1964). The fractionated classes were detected by exposing the plates to iodine vapour or by charring at $220^{\circ} \mathrm{C}$ after spraying the plates with $50 \%(\mathrm{w} / \mathrm{v}) \mathrm{H}_{2} \mathrm{SO}_{4}$. The lipid classes were identified by comparing their chromatographic behaviour with that of authentic samples and by the colour reactions they gave with specific spray reagents (Stahl, 1962; Dittmer \& Lester, 1964; Siakotos \& Rouser, 1965). Lipid classes in $1 \mathrm{mg}$ total lipids were resolved by TLC and determined quantitatively by GLC of their acyl moieties, after methanolysis (Chalvardjian, 1964), using heptadecanoic acid as an internal standard (Radwan, 1978). The fatty acid methyl esters were analysed using a Pye-Unicam 204 gas-chromatograph fitted with a glass column, $1.83 \mathrm{~m} \times 4 \mathrm{~mm}$ i.d., packed with $15 \%$ (v/v) diethyleneglycol succinate (DEGS) on Anakrom D, 100-120 mesh. Individual fatty acids were identified by comparing their retention times with those of standard samples. The identity of arachidonic acid $(20: 4)$ was confirmed by combined argentation TLC and GLC. The tetraenoic methyl ester fraction, which cochromatographed with standard methyl arachidonate on $10 \%(\mathrm{w} / \mathrm{w}) \mathrm{AgNO}_{3}$ impregnated silica gel plates using hexane/diethyl ether $(80: 20, \mathrm{v} / \mathrm{v})$ as a developing solvent, was scraped off and the methyl esters were eluted (Kates, 1972) and analysed by GLC.

\section{RESULTS}

\section{Fatty acid biodegradation}

Table 1 presents data on the biodegradation of fatty acids and sodium salts of fatty acids added to fresh soil suspensions. In all cases, the sodium salts of fatty acids disappeared more readily than the free acids. Fatty acids and the sodium salts of fatty acids that had been added to sterile soil suspensions were recovered quantitatively after 24 weeks.

\section{Fatty acid utilizing micro-organisms}

Direct analysis of fresh desert and garden soil samples showed that they were poor in fatty acid utilizing micro-organisms. However, soil suspensions supplemented with fatty acids or the sodium salts of fatty acids became, after 24 weeks, enriched with fungi capable of utilizing these compounds as sole sources of carbon and energy. The total numbers ranged between $3.0 \times 10^{3}$ $( \pm 156)$ and $3.9 \times 10^{4}( \pm 282)$ propagules $\mathrm{ml}^{-1}$. These fungi are listed in Table 2 . Neither bacteria nor actinomycetes were recorded.

All the fungi listed in Table 2 were unable to grow on the basal medium without the respective fatty acids or their sodium salts.

Eleven isolates were selected for further study, on the basis of showing good growth (in preliminary experiments) on the fatty acids: 14:0 (Aspergillus versicolor and Aspergillus oryzae); 16:0 (Aspergillus ustus, Aspergillus fumigatus and Paecilomyces lilacinus); 18:0 (Fusarium oxysporum and Fusarium sp.); and 18:1 (Aspergillus niger, A. ustus, Paec. lilacinus and Penicillium sp.). Utilizers of $8: 0$ showed only poor growth, hence they were not studied further. The optimal concentration of fatty acids and their salts for the growth of all fungi was $10 \mathrm{~g} \mathrm{l}^{-1}$. None of the 11 isolates showed strict specificity to the fatty acid or sodium salt of the fatty acid on which it had been isolated. All fungi grew when supplied with $14: 0,16: 0,18: 0$ or $18: 1$ as either free acids or 
Table 1. Biodegradation of fatty acids and sodium salts of fatty acids in soil suspensions Data represent the means \pm SD of three determinations.

Percentage loss after:

\section{Compounds*}

Caprylic acid

Sodium caprylate

Myristic acid

Sodium myristate

Palmitic acid

Sodium palmitate

Stearic acid

Sodium stearate

Oleic acid

Sodium oleate

\begin{tabular}{|c|c|}
\hline \multicolumn{2}{|c|}{12 weeks } \\
\hline Desert & Garden \\
\hline $36 \cdot 2 \pm 3 \cdot 6$ & $74.4 \pm 3.0$ \\
\hline $2.8 \pm 6.0$ & $95 \cdot 0 \pm 2 \cdot 3$ \\
\hline $31 \cdot 0 \pm 4 \cdot 0$ & $50.0 \pm 2.6$ \\
\hline $93.9 \pm 5.2$ & $91.4 \pm 4.4$ \\
\hline $36.5 \pm 2.3$ & $45.0 \pm 0.9$ \\
\hline $8 \cdot 8 \pm 1 \cdot 2$ & $91 \cdot 3 \pm 5.5$ \\
\hline $71 \cdot 3 \pm 4 \cdot 0$ & $68 \cdot 5 \pm 1 \cdot 2$ \\
\hline $73 \cdot 8 \pm 2 \cdot 6$ & $91 \cdot 1 \pm 4 \cdot 6$ \\
\hline $31 \cdot 4 \pm 3 \cdot 1$ & $64 \cdot 5 \pm 1 \cdot 1$ \\
\hline $96 \cdot 2 \pm$ & $94 \cdot 5 \pm 3 \cdot 7$ \\
\hline
\end{tabular}

\begin{tabular}{|c|c|}
\hline \multicolumn{2}{|c|}{24 weeks } \\
\hline Desert & Garden \\
\hline $90 \cdot 0 \pm 5 \cdot 5$ & $95 \cdot 3 \pm 4 \cdot 2$ \\
\hline $100 \cdot 0$ & $100 \cdot 0$ \\
\hline $97 \cdot 8 \pm 1 \cdot 1$ & $97 \cdot 5 \pm 2 \cdot 3$ \\
\hline $100 \cdot 0$ & $100 \cdot 0$ \\
\hline $98.0 \pm 0.5$ & $91 \cdot 3 \pm 5.9$ \\
\hline $100 \cdot 0$ & $100 \cdot 0$ \\
\hline $99.8 \pm 0.2$ & $82 \cdot 6 \pm 1 \cdot 4$ \\
\hline $100 \cdot 0$ & $100 \cdot 0$ \\
\hline $95 \cdot 3 \pm 3 \cdot 1$ & $94 \cdot 0 \pm 2 \cdot 9$ \\
\hline $100 \cdot 0$ & $100 \cdot 0$ \\
\hline
\end{tabular}

- All compounds were recovered quantitatively from sterile soil suspensions after 24 weeks.

Table 2. Soil fungi utilizing individual fatty acids as sole sources of carbon and energy

Desert soil suspensions treated with:

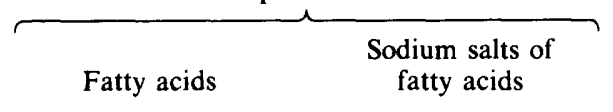

$8: 0$ utilizers

Fusarium sp.

Fusarium sp.

14:0 utilizers

Penicillium cyclopium Aspergillus versicolor

Aspergillus ustus

Cladosporium herbarum Fusarium oxysporum

16:0 utilizers

Penicillium cyclopium

Aspergillus fumigatus

18:0 utilizers Aspergillus flavus Aspergillus fumigatus

Penicillium chrysogenum Aspergillus flavus Aspergillus versicolor Aspergillus ficuum Aspergillus fumigatus

Aspergillus versicolor Trichoderma koningii

Fusarium oxysporum Aspergillus terricola Penicillium cyclopium

$18: 1$ utilizers Aspergillus niger Trichoderma viride
Paecilomyces lilacinus Penicillium sp.
Garden soil suspensions treated with:

Fatty acids

Sodium salts of fatty acids

Fusarium sp.

Aspergillus sp. Fusarium sp.

\section{Trichoderma koningii Aspergillus oryzae Aspergillus ficuum Aspergillus japonicus Cladosporium herbarum Aspergillus niger Stachybotrys sp.}

Aspergillus flavus Trichoderma koningii Paecilomyces lilacinus Cladosporium herbarum

Aspergillus ustus Aspergillus japonicus Penicillium italicum

Fusarium oxysporum

\section{Aspergillus fumigatus} Cladosporium sp. Fusarium moniliforme
Fusarium solani Fusarium sp.

\author{
Penicillium chrysogenum \\ Aspergillus flavus \\ Aspergillus wentii \\ Aspergillus fumigatus \\ Fusarium sp.
}

as their sodium salts. They also grew well on glucose; with the exception of $A$. niger the various isolates grew poorly, if at all, on citric acid (Table 3 ).

Many isolates could grow over a wide range of $\mathrm{pH}$, tolerating high acidity better than high alkalinity, with their optima between pH 6 and 8 (Fig. 1). This experiment was done with the water soluble sodium salts of the fatty acids but at acidic $\mathrm{pH}$ values the compounds occurred as the water insoluble free acids. 

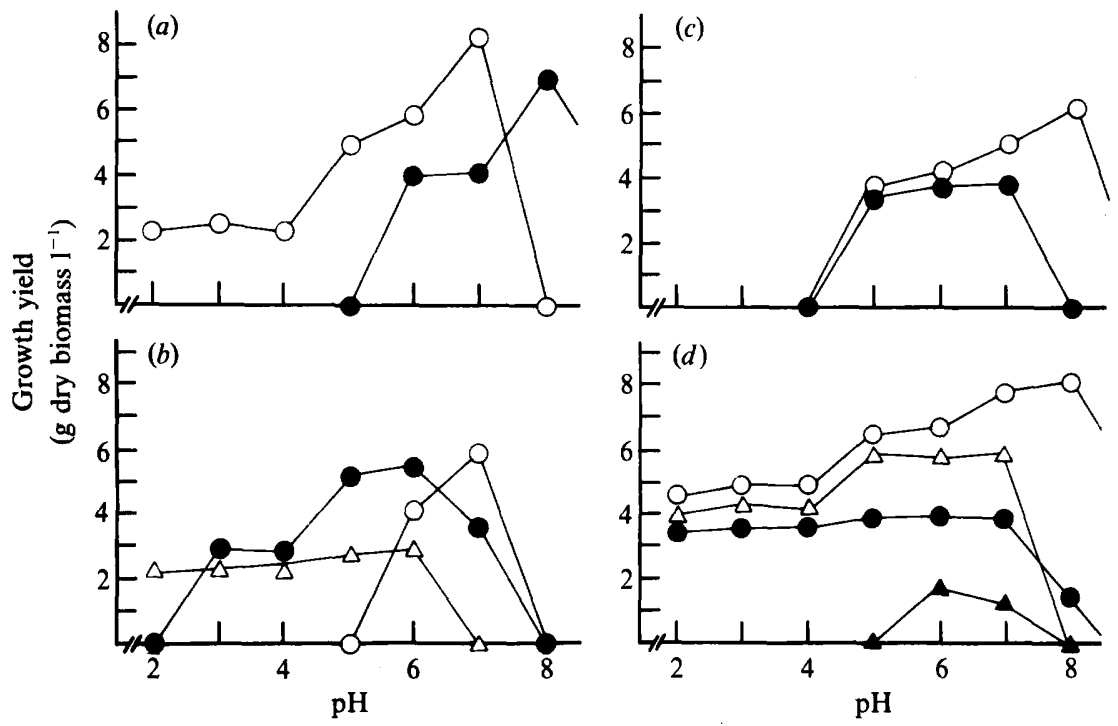

Fig. 1. Effect of $\mathrm{pH}$ on the growth yield of fatty acid utilizing fungi. (a) 14:0 utilizers: $A$. oryzae (O); $A$. versicolor (O). (b) 16:0 utilizers: A. ustus $(O) ;$ Paec. lilacinus $(O) ; A$. fumigatus $(\triangle)$. (c) 18:0 utilizers: F.oxysporum (O); Fusarium sp. (O). (d) 18:1 utilizers: Penicillium sp. (O); Paec. lilacinus $(\bigcirc)$; $A$.niger $(\triangle) ;$ A. ustus (A).

Table 3. Growth of fatty acid utilizing fungi on different carbon sources

Data represent the means \pm SD of three experiments. The concentrations of the various carbon sources added to the media were calculated so as to provide equal amounts of carbon.

\begin{tabular}{|c|c|c|c|}
\hline & \multicolumn{3}{|c|}{ Growth [g dry biomass $(1 \text { medium })^{-1}$ ] } \\
\hline & $\begin{array}{l}\text { Fatty acids } \\
\left(10 \mathrm{~g}^{-1}\right)\end{array}$ & $\begin{array}{c}\text { Glucose } \\
\left(18 \mathrm{~g} \mathrm{I}^{-1}\right)\end{array}$ & $\begin{array}{l}\text { Citric acid } \\
\left(20 \mathrm{~g}^{-1}\right)\end{array}$ \\
\hline $\begin{array}{l}\text { 14:0 utilizers } \\
\text { Aspergillus versicolor } \\
\text { Aspergillus oryzae }\end{array}$ & $\begin{array}{l}2 \cdot 3 \pm 0 \cdot 3 \\
4 \cdot 0 \pm 0 \cdot 2\end{array}$ & $\begin{array}{l}2 \cdot 8 \pm 0.2 \\
2 \cdot 8 \pm 0.3\end{array}$ & $\begin{array}{l}0 \cdot 8 \pm 0 \cdot 1 \\
0\end{array}$ \\
\hline $\begin{array}{l}16: 0 \text { utilizers } \\
\text { Aspergillus ustus } \\
\text { Aspergillus fumigatus } \\
\text { Paecilomyces lilacinus }\end{array}$ & $\begin{array}{l}1.9 \pm 0.1 \\
2.9 \pm 0.4 \\
3.6 \pm 0.2\end{array}$ & $\begin{array}{l}2 \cdot 6 \pm 0 \cdot 1 \\
2 \cdot 2 \pm 0 \cdot 1 \\
2 \cdot 2 \pm 0 \cdot 2\end{array}$ & $\begin{array}{l}0 \\
0 \\
0 \cdot 3 \pm 0\end{array}$ \\
\hline $\begin{array}{l}\text { 18:0 utilizers } \\
\text { Fusarium oxysporum } \\
\text { Fusarium sp. }\end{array}$ & $\begin{array}{l}1 \cdot 9 \pm 0 \\
2 \cdot 6 \pm 0 \cdot 1\end{array}$ & $\begin{array}{l}2 \cdot 1 \pm 0.2 \\
1 \cdot 8 \pm 0.0\end{array}$ & $\begin{array}{l}0 \\
0.5 \pm 0\end{array}$ \\
\hline $\begin{array}{l}\text { 18:1 utilizers } \\
\text { Aspergillus niger } \\
\text { Aspergillus ustus } \\
\text { Paecilomyces lilacinus } \\
\text { Penicillium } \mathrm{sp} .\end{array}$ & $\begin{array}{l}1 \cdot 8 \pm 0 \cdot 1 \\
2 \cdot 1 \pm 0 \cdot 3 \\
1 \cdot 5 \pm 0 \cdot 1 \\
1 \cdot 3 \pm 0\end{array}$ & $\begin{array}{l}2.7 \pm 0.1 \\
2 \cdot 3 \pm 0.1 \\
2.6 \pm 0.2 \\
2.4 \pm 0.1\end{array}$ & $\begin{array}{l}2 \cdot 0 \pm 0 \cdot 1 \\
0 \\
0 \cdot 4 \pm 0 \\
0 \cdot 4 \pm 0 \cdot 1\end{array}$ \\
\hline
\end{tabular}

\section{Lipid composition of fungi}

Analysis by TLC showed that in lipid extracts of the 11 fungi grown on fatty acids, but not on glucose, the free fatty acids were the predominant lipid class (Fig. 2) making up $60 \cdot 7 \%-91 \cdot 4 \%$ (on glucose only $12 \cdot 7 \%-21 \cdot 1 \%$ ) of the total lipids. In addition, considerable proportions of triacylglycerols, sterols, phosphatidylcholines and phosphatidylethanolamines were present. Small amounts of phosphatidyl glycerols, phosphatidyl inositols, phosphatidyl serines, ceramide monohexosides and a few unidentified polar compounds were also present in all extracts. 


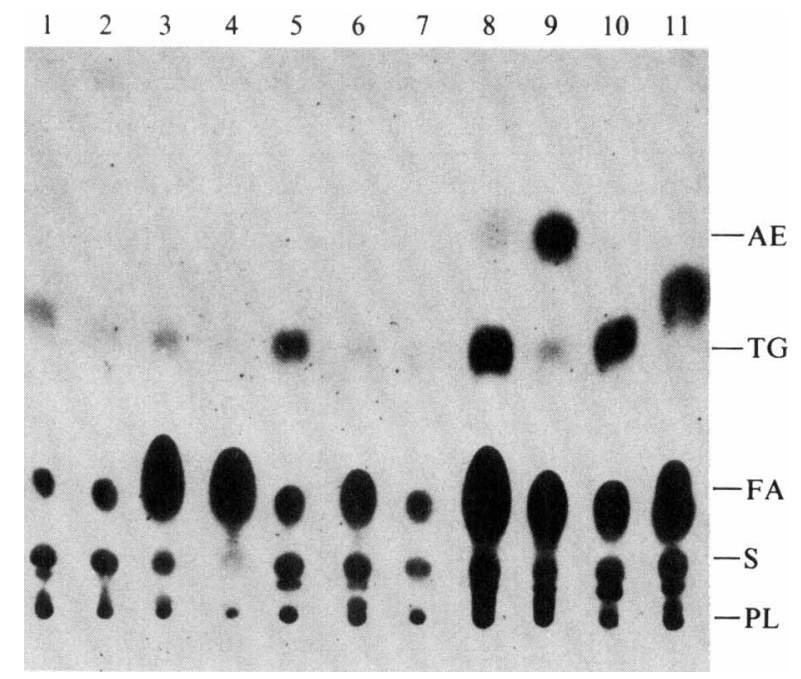

Fig. 2. Typical TLC plate showing the lipid composition of fatty acid utilizing fungi. The sorbent was Silica Gel G; the solvent was hexane/diethyl ether/acetic acid ( $90: 10: 1$, by vol.). The lipids were visualized by charring. AE, alkyl esters; TG, triacylglycerols; FA, fatty acids; S, sterols; PL, polar lipids. 14:0 utilizers: $A$. versicolor (lane 1); $A$. oryzae (lane 2). 16:0 utilizers: $A$. ustus (lane 3 ); $A$. fumigatus (lane 4); Paec. lilacinus (lane 5). 18:0 utilizers: F. oxysporum (lane 6); Fusarium sp. (lane 7). 18:1 utilizers: A. ustus (lane 8); A. niger (lane 9); Paec. lilacinus (lane 10); Penicillium sp. (lane 11).

\section{Constituent faty acids of predominant lipid classes}

The fatty acid profiles of the four major lipid classes in the total lipid from the 11 fungi grown on fatty acids are shown in Table 4. For comparison the fatty acid patterns of the same lipid classes from four representative fungi grown on glucose are also included in this table. It is obvious that the fatty acid patterns varied for the same fungus according to whether it had utilized glucose or a fatty acid. Lipid classes from glucose utilizing fungi contained predominantly 16:0,18:0,18:1 and linoleic $(18: 2)$ acids. Only small amounts of linolenic $(18: 3)$ and arachidonic $(20: 4)$ acids were present. Lipid classes from the various fungi usually contained variable concentrations of the fatty acids on which they grew. The $16: 0$ utilizing fungi accumulated relatively large amounts of linolenic acid $(18: 3)$ in their phospholipids. In most cases, fungi grown on fatty acids contained in their lipid classes relatively more $20: 4$ than in the same lipid classes from the same fungi grown on glucose. The highest concentrations of $20: 4$ in the major class of fatty acids were recorded in fungi utilizing fatty acids with the shortest chains, namely in the 14:0 utilizer $A$. versicolor and the 16:0 utilizer $A$. ustus. The identity of this polyunsaturated long-chain fatty acid was confirmed using argentation TLC and GLC as described above.

\section{DISCUSSION}

Fatty acid utilizing micro-organisms may provide a means of recycling wastes such as waste soap in sewage, since soap consists of fatty acid salts. These studies can also contribute to our understanding of petroleum biodegradation in view of the fact that aliphatic hydrocarbons are first oxidized to the corresponding fatty acids before they are biodegraded (e.g. Alexander, 1977).

The present paper reports on soil fungi, predominantly Aspergillus spp., that are capable of utilizing $C_{14}, C_{16}$ and $C_{18}$ fatty acids as sole sources of carbon and energy. Fatty acids with shorter chains do not seem to be suitable substrates for microbial nutrition. It is interesting that the growth yields of fungi on fatty acids were comparable with their growth yields on the conventional carbon source glucose. In our experiments the insoluble fatty acids could be utilized by the fungi only at the relatively small lipid-aqueous-phase interface. It may be expected that more utilization could occur if the fatty acids were emulsified in the medium. The growth of fungi on fatty acids suggests that these compounds are first degraded into $\mathrm{C}_{2}$ units, which are used, through the citric acid cycle, both for energy production and biosynthesis of 
Table 4. Fatty acid patterns of major lipid classes from fungi grown on fatty acids or glucose Values are expressed as percentages and are the means of two determinations.

\begin{tabular}{|c|c|c|c|c|c|c|c|c|c|c|}
\hline \multirow{3}{*}{$\begin{array}{l}\text { Fatty } \\
\text { acid }\end{array}$} & \multirow{3}{*}{$\begin{array}{l}\text { Lipid } \\
\text { classis }^{*}\end{array}$} & \multirow{3}{*}{$\begin{array}{c}\text { Fungus... } \\
\text { Carbon } \\
\text { source.. }\end{array}$} & \multicolumn{3}{|c|}{$14: 0$ utilizers } & \multicolumn{4}{|c|}{$16: 0$ utilizers } & \\
\hline & & & \multicolumn{2}{|c|}{ A. versicolor } & \multirow{2}{*}{$\begin{array}{c}\text { A. orizae } \\
14: 0\end{array}$} & \multicolumn{2}{|c|}{ Paec. lilacinus } & \multirow{2}{*}{$\begin{array}{r}\text { A. ustus } \\
16: 0\end{array}$} & \multirow{2}{*}{$\begin{array}{l}\text { A. fumigatus } \\
\qquad 16: 0\end{array}$} & \\
\hline & & & Glucose & $14: 0$ & & Glucose & $16: 0$ & & & \\
\hline $14: 0$ & $\begin{array}{l}\text { FA } \\
\text { TG } \\
\text { PC } \\
\text { PE }\end{array}$ & & $\begin{array}{l}1 \cdot 1 \\
\text { tr. } \\
\text { tr. } \\
\text { tr. }\end{array}$ & $\begin{array}{r}8.5 \\
14.8 \\
10.8 \\
6.7\end{array}$ & $\begin{array}{r}3.0 \\
11 \cdot 1 \\
4.7 \\
9 \cdot 0\end{array}$ & $\begin{array}{l}\text { tr. } \\
0 \cdot 2 \\
\text { tr. } \\
2 \cdot 5\end{array}$ & $\begin{array}{l}0.9 \\
1.5 \\
1.2 \\
2.0\end{array}$ & $\begin{array}{l}8 \cdot 1 \\
0.9 \\
2 \cdot 1 \\
1.9\end{array}$ & $\begin{array}{l}0 \cdot 2 \\
3 \cdot 0 \\
2 \cdot 8 \\
1 \cdot 2\end{array}$ & \\
\hline $16: 0$ & $\begin{array}{l}\text { FA } \\
\text { TG } \\
\text { PC } \\
\text { PE }\end{array}$ & & $\begin{array}{l}14 \cdot 3 \\
14 \cdot 3 \\
22 \cdot 5 \\
26 \cdot 1\end{array}$ & $\begin{array}{r}13.6 \\
16.6 \\
15.8 \\
6.7\end{array}$ & $\begin{array}{r}14 \cdot 3 \\
6 \cdot 2 \\
5 \cdot 8 \\
6 \cdot 4\end{array}$ & $\begin{array}{l}18 \cdot 0 \\
26 \cdot 6 \\
25 \cdot 4 \\
28 \cdot 6\end{array}$ & $\begin{array}{l}69 \cdot 9 \\
58 \cdot 3 \\
14 \cdot 8 \\
29 \cdot 6\end{array}$ & $\begin{array}{l}12 \cdot 6 \\
87.6 \\
19 \cdot 8 \\
15 \cdot 4\end{array}$ & $\begin{array}{l}97 \cdot 6 \\
18 \cdot 3 \\
12.4 \\
36.9\end{array}$ & \\
\hline $18: 0$ & $\begin{array}{l}\text { FA } \\
\text { TG } \\
P, C \\
P . E\end{array}$ & & $\begin{array}{r}13 \cdot 9 \\
24 \cdot 3 \\
5 \cdot 4 \\
5 \cdot 6\end{array}$ & $\begin{array}{c}4.5 \\
4.8 \\
\text { tr. } \\
1.6\end{array}$ & $\begin{array}{r}13 \cdot 6 \\
3.6 \\
2.8 \\
1.8\end{array}$ & $\begin{array}{r}10 \cdot 9 \\
16.9 \\
5.5 \\
10 \cdot 1\end{array}$ & $\begin{array}{c}10 \cdot 4 \\
6 \cdot 7 \\
\text { tr. } \\
12 \cdot 4\end{array}$ & $\begin{array}{l}4 \cdot 0 \\
3 \cdot 4 \\
9 \cdot 6 \\
\text { tr. }\end{array}$ & $\begin{array}{l}1 \cdot 1 \\
7.5 \\
\operatorname{tr} . \\
1 \cdot 4\end{array}$ & \\
\hline $18: 1$ & $\begin{array}{l}\text { 䎡A } \\
\text { TG } \\
\text { PC } \\
\text { PE } \\
\text { FA } \\
\text { TG } \\
\text { PC } \\
\text { PE }\end{array}$ & & $\begin{array}{l}20.1 \\
31.9 \\
18.0 \\
22.3 \\
42.8 \\
25.8 \\
45.0 \\
45.9\end{array}$ & $\begin{array}{r}5 \cdot 1 \\
24.7 \\
9.1 \\
10 \cdot 3 \\
12.2 \\
20.8 \\
28.4 \\
24 \cdot 0\end{array}$ & $\begin{array}{r}42 \cdot 1 \\
5.2 \\
20.5 \\
7.2 \\
9 \cdot 0 \\
15 \cdot 0 \\
21 \cdot 7 \\
33 \cdot 4\end{array}$ & $\begin{array}{l}27.3 \\
32.5 \\
21.4 \\
16.8 \\
43.7 \\
22.9 \\
43.4 \\
18.5\end{array}$ & $\begin{array}{r}10.8 \\
19.7 \\
17.8 \\
16.4 \\
2.6 \\
8.3 \\
32.9 \\
12.9\end{array}$ & $\begin{array}{r}7.1 \\
1.8 \\
12.9 \\
11.1 \\
8.1 \\
0.6 \\
13.8 \\
14.6\end{array}$ & $\begin{array}{c}0.2 \\
10.7 \\
10.8 \\
11.9 \\
\text { tr. } \\
3.7 \\
\text { tr. } \\
\text { tr. }\end{array}$ & \\
\hline $18: 3$ & $\begin{array}{l}\text { FA } \\
\text { TG } \\
\text { PC } \\
\text { PE }\end{array}$ & & $\begin{array}{l}\text { tr. } \\
1 \cdot 9 \\
\text { tr. } \\
\text { tr. }\end{array}$ & $\begin{array}{l}10.9 \\
\text { tr. } \\
\text { tr. } \\
\text { tr. }\end{array}$ & $\begin{array}{l}\text { tr. } \\
4 \cdot 5 \\
\text { tr. } \\
1 \cdot 7\end{array}$ & $\begin{array}{l}\text { tr. } \\
0.9 \\
\text { tr. } \\
\text { tr. }\end{array}$ & $\begin{array}{c}\text { tr. } \\
\text { tr. } \\
18 \cdot 0 \\
19 \cdot 1\end{array}$ & $\begin{array}{l}\text { tr. } \\
\text { tr. } \\
35 \cdot 6 \\
41 \cdot 1\end{array}$ & $\begin{array}{c}\text { tr. } \\
0 \cdot 3 \\
53.9 \\
41 \cdot 0\end{array}$ & \\
\hline \multirow[t]{2}{*}{$20: 4$} & \multirow[t]{2}{*}{$\begin{array}{l}\text { FA } \\
\text { TG } \\
\text { PC } \\
P E\end{array}$} & & $\begin{array}{l}2 \cdot 1 \\
0 \cdot 5 \\
9 \cdot 0 \\
\text { tr. }\end{array}$ & $\begin{array}{l}29 \cdot 4 \\
15 \cdot 7 \\
23 \cdot 7 \\
28 \cdot 8\end{array}$ & $\begin{array}{c}\text { tr. } \\
1.8 \\
12.6 \\
20.8\end{array}$ & $\begin{array}{c}\text { tr. } \\
\text { tr. } \\
3 \cdot 3 \\
16 \cdot 8\end{array}$ & $\begin{array}{r}2 \cdot 3 \\
4 \cdot 4 \\
14 \cdot 1 \\
5 \cdot 8\end{array}$ & $\begin{array}{r}26.9 \\
0.9 \\
3.9 \\
12.8\end{array}$ & $\begin{array}{r}0 \cdot 2 \\
24 \cdot 2 \\
12 \cdot 1 \\
2 \cdot 5\end{array}$ & \\
\hline & & & \multicolumn{3}{|c|}{$18: 0$ utilizers } & \multicolumn{5}{|c|}{$18: 1$ utilizers } \\
\hline \multirow{2}{*}{$\begin{array}{l}\text { Fatty } \\
\text { acid }\end{array}$} & \multirow{2}{*}{$\begin{array}{l}\text { Lipid } \\
\text { class* }\end{array}$} & \multirow{2}{*}{$\begin{array}{c}\text { Fungus... } \\
\text { Carbon } \\
\text { source .. }\end{array}$} & \multicolumn{2}{|c|}{ F. oxysporum } & \multirow{2}{*}{$\begin{array}{c}\text { Fusarium sp. } \\
18: 0\end{array}$} & \multicolumn{2}{|c|}{$\underbrace{A .}$ niger } & A. ustus & \multirow{2}{*}{$\begin{array}{c}\text { Paec. } \\
\text { lilacinus } \\
18: 1\end{array}$} & Penicillium sp \\
\hline & & & Glucose & $18: 0$ & & Glucose & $18: 1$ & $18: 1$ & & $18: 1$ \\
\hline $14: 0$ & $\begin{array}{l}\text { FA } \\
\text { TG } \\
\text { PC } \\
\text { PE }\end{array}$ & & $\begin{array}{c}1 \cdot 3 \\
1.0 \\
\text { tr.. } \\
0.5\end{array}$ & $\begin{array}{l}1 \cdot 0 \\
3 \cdot 1 \\
\text { tr. } \\
\text { tr. }\end{array}$ & $\begin{array}{r}1.6 \\
11.7 \\
6.6 \\
4.2\end{array}$ & $\begin{array}{l}\text { tr. } \\
1.7 \\
\text { tr. } \\
5.4\end{array}$ & $\begin{array}{l}2 \cdot 8 \\
4 \cdot 8 \\
\text { tr. } \\
\text { tr. }\end{array}$ & $\begin{array}{c}0 \cdot 7 \\
4 \cdot 0 \\
\text { tr. } \\
0 \cdot 7\end{array}$ & $\begin{array}{l}1 \cdot 3 \\
3 \cdot 5 \\
\text { tr. } \\
\text { tr. }\end{array}$ & $\begin{array}{l}0 \cdot 3 \\
\text { tr. } \\
\text { tr. } \\
\text { tr. }\end{array}$ \\
\hline $16 \vdots 0$ & $\begin{array}{l}\text { FA } \\
\text { TG } \\
\text { PC } \\
\text { PE }\end{array}$ & & $\begin{array}{l}46 \cdot 9 \\
16 \cdot 7 \\
10 \cdot 2 \\
19 \cdot 9\end{array}$ & $\begin{array}{r}23 \cdot 1 \\
9 \cdot 2 \\
4 \cdot 5 \\
31.8\end{array}$ & $\begin{array}{r}9 \cdot 6 \\
7.3 \\
25.9 \\
7.5\end{array}$ & $\begin{array}{l}47 \cdot 3 \\
47 \cdot 2 \\
23 \cdot 0 \\
15 \cdot 3\end{array}$ & $\begin{array}{l}16.0 \\
11.4 \\
13.8 \\
21.1\end{array}$ & $\begin{array}{r}2.8 \\
10.5 \\
7.9 \\
15.9\end{array}$ & $\begin{array}{r}7 \cdot 6 \\
6 \cdot 7 \\
10 \cdot 2 \\
13 \cdot 2\end{array}$ & $\begin{array}{l}51.8 \\
10.9 \\
11.8 \\
16.1\end{array}$ \\
\hline $18: 0$ & $\begin{array}{l}\text { FA } \\
\text { TG } \\
\text { PC } \\
\text { PE }\end{array}$ & & $\begin{array}{r}22 \cdot 3 \\
16.7 \\
3 \cdot 6 \\
4 \cdot 3\end{array}$ & $\begin{array}{r}22.5 \\
28.3 \\
3.8 \\
18.5\end{array}$ & $\begin{array}{r}42.5 \\
12.6 \\
13.9 \\
5.0\end{array}$ & $\begin{array}{c}12 \cdot 1 \\
8 \cdot 7 \\
\text { tr. } \\
15 \cdot 3\end{array}$ & $\begin{array}{r}29.8 \\
3.2 \\
10.6 \\
5.6\end{array}$ & $\begin{array}{l}7 \cdot 1 \\
4.8 \\
1.5 \\
3.6\end{array}$ & $\begin{array}{c}18.7 \\
4.8 \\
\text { tr. } \\
3.8\end{array}$ & $\begin{array}{r}11.7 \\
5.7 \\
2.1 \\
6.8\end{array}$ \\
\hline $18: 1$ & $\begin{array}{l}\text { FA } \\
\text { TG } \\
\text { PC } \\
\text { PE }\end{array}$ & & $\begin{array}{r}7.0 \\
19 \cdot 5 \\
5.2 \\
4.9\end{array}$ & $\begin{array}{r}44.4 \\
3.9 \\
4 \cdot 8 \\
10.1\end{array}$ & \begin{tabular}{r}
\multicolumn{1}{c}{ tr. } \\
3.8 \\
$21 \cdot 9$ \\
$8 \cdot 3$
\end{tabular} & $\begin{array}{l}22 \cdot 5 \\
23 \cdot 2 \\
28 \cdot 8 \\
15 \cdot 3\end{array}$ & $\begin{array}{l}28.9 \\
35.6 \\
35.9 \\
35.4\end{array}$ & $\begin{array}{l}45 \cdot 3 \\
24 \cdot 0 \\
21 \cdot 2 \\
23 \cdot 7\end{array}$ & $\begin{array}{l}51 \cdot 2 \\
52.9 \\
48.9 \\
33.0\end{array}$ & $\begin{array}{l}28.7 \\
24.5 \\
15.9 \\
18.4\end{array}$ \\
\hline $18: 2$ & $\begin{array}{l}\text { FA } \\
\text { TG } \\
\text { PC } \\
\text { PE }\end{array}$ & & $\begin{array}{l}15.9 \\
40 \cdot 5 \\
69 \cdot 3 \\
58.2\end{array}$ & $\begin{array}{r}2.6 \\
2.3 \\
18.8 \\
21.7\end{array}$ & $\begin{array}{l}2 \cdot 1 \\
6 \cdot 6 \\
4 \cdot 8 \\
4.9\end{array}$ & $\begin{array}{l}18.1 \\
15.7 \\
46.0 \\
15.9\end{array}$ & $\begin{array}{r}1.6 \\
5.6 \\
14.8 \\
15.5\end{array}$ & $\begin{array}{r}1.7 \\
5.5 \\
57.4 \\
28.4\end{array}$ & $\begin{array}{r}2.9 \\
12.5 \\
30.6 \\
15.7\end{array}$ & $\begin{array}{r}2 \cdot 5 \\
19 \cdot 6 \\
47 \cdot 2 \\
42 \cdot 7\end{array}$ \\
\hline $18: 3$ & $\begin{array}{l}\text { FA } \\
\text { TG } \\
\text { PC } \\
\text { PE }\end{array}$ & & $\begin{array}{l}0.8 \\
1.5 \\
9.2 \\
4.7\end{array}$ & $\begin{array}{l}2 \cdot 2 \\
\text { tr. } \\
\text { tr. } \\
\text { tr. }\end{array}$ & $\begin{array}{l}\text { tr. } \\
\text { tr. } \\
6.6 \\
12.7\end{array}$ & $\begin{array}{l}\text { tr. } \\
\text { tr. } \\
\text { tr. } \\
\text { tr. }\end{array}$ & $\begin{array}{l}11 \cdot 1 \\
\text { tr. } \\
\text { tr. } \\
\text { tr. }\end{array}$ & $\begin{array}{l}\text { tr. } \\
\text { tr. } \\
\text { tr. } \\
\text { tr. }\end{array}$ & $\begin{array}{l}\text { tr. } \\
13 \cdot 0 \\
\text { tr. } \\
\text { tr. }\end{array}$ & $\begin{array}{l}\text { tr. } \\
\text { tr. } \\
\text { tr. } \\
1 \cdot 2\end{array}$ \\
\hline $20: 4$ & $\begin{array}{l}\text { FA } \\
\text { TG } \\
\text { PC } \\
\text { PE }\end{array}$ & & $\begin{array}{l}1.6 \\
2.8 \\
1.0 \\
5.4\end{array}$ & $\begin{array}{r}2.9 \\
8.7 \\
22.6 \\
8.7\end{array}$ & $\begin{array}{r}5.3 \\
1.8 \\
4.0 \\
23.6\end{array}$ & $\begin{array}{c}\text { tr. } \\
2.9 \\
\text { tr. } \\
15 \cdot 3\end{array}$ & $\begin{array}{r}1.5 \\
23.8 \\
17.2 \\
22.4\end{array}$ & $\begin{array}{r}1.0 \\
24.0 \\
7.3 \\
13.7\end{array}$ & \begin{tabular}{r}
\multicolumn{1}{c}{ tr. } \\
6.5 \\
10.2 \\
$5 \cdot 3$
\end{tabular} & $\begin{array}{r}3 \cdot 8 \\
16 \cdot 3 \\
4 \cdot 2 \\
12 \cdot 0\end{array}$ \\
\hline
\end{tabular}

* FA, fatty acids; TG, triacylglycerols; PC, phosphatidylcholines; PE, phosphatidylethanolamines. tr., Trace. 
cellular material. In the lipid extracts of all the fungi tested, free fatty acids were by far the most predominant. This was not the case for fungi grown on glucose. This high content of fatty acids is not artificial since lipases were inactivated before and during extraction. This result indicates that the mycelia actively accumulate fatty acids from the nutrient medium. Other biological systems, such as plant cell cultures, contain in the cell walls acyl carrier proteins that accumulate fatty acids from the media (for a review see Radwan \& Mangold, 1980). However, in most of the fungi the predominant fatty acid was not the one added to the growth medium. Mycelial fatty acids were apparently synthesized anew from $\mathrm{C}_{2}$ units derived from the utilized fatty acids. Some of the absorbed fatty acids may have been directly modified, e.g. some 16:0 appears to have been desaturated and elongated to $18: 3$, which, in turn, was mainly incorporated into phospholipids. On the other hand, neither of the two $\mathrm{C}_{18}$ fatty acids seemed to have been elongated or desaturated. This becomes particularly apparent when we compare the fatty acid patterns of lipids from A. ustus and Paec. lilacinus grown on 16:0 with fatty acid patterns of lipids from the same fungi grown on 18:1. On 16:0 the phospholipids from both fungi were rich in $18: 3$, whereas on $18: 1$ they were poor in this polyunsaturated fatty acid.

Irrespective of how the utilized fatty acids are further metabolized it is apparent that certain utilizers of short-chain fatty acids (14:0 and 16:0) have the potential of producing high levels of $20: 4$. This fatty acid, which is a useful precursor of prostaglandins (Pace-Asciak \& Wolfe, 1971; Horton, 1972) is obtained mainly from animals; its production via micro-organisms is recommended (Korn et al., 1965; Iizuka et al., 1979).

Fungal isolates were identified in the 'Deutsche Sammlung von Mikroorganismen' in Göttingen, FRG, in collaboration with P. Hoffmann and lipids were analysed in the 'Bundesanstalt für Fettforschung' in Münster, FRG, in collaboration with H. K. Mangold.

\section{REFERENCES}

AleXANDER, M. (1977). Introduction to Soil Microbiology, 2nd edn. New York: John Wiley.

Booth, C. (1971). The Genus Fusarium, Kew, Surrey, UK: Commonwealth Mycological Institute.

Chalvardian, A. (1964). Fatty acids of brown and yellow fat in rats. Biochemical Journal 90, 518-521.

Christie, W. W. (1973). Lipid Analysis. New York: Pergamon Press.

DITTMER, J.C. \& LESTER, R. L. (1964). A simple specific spray for the detection of phospholipids on thin-layer chromatograms. Journal of Lipid Research 5, 126-127.

Domasch, K. H., Gams, W. \& Anderson, T. H. (1980). Compendium of Soil Fungi. London: Academic Press.

Folch, J., Lees, M. \& Sloane-Stanley, G. H. (1957). A simple method for the isolation and purification of total lipids from animal tissues. Journal of Biological Chemistry 226, 497-509.

HaRP, E. F. (1979). Environmental Problems. In Fatty Acids, pp. 90-109. Edited by E. H. Pryde. Champaign, Illinois: American Oil Chemists Society.

Horton, E. W. (1972). Prostaglandins, pp. 1-64, Berlin: Springer.

IIzUKA, H., Онтомо, T. \& Yoshid, K. (1979). Production of arachidonic acid by a hydrocarbonutilizing strain of Penicillium cyaneum. European Journal of Applied Microbiology and Biotechnology 7, 173-180.

KATES, M. (1972). Techniques of Lipidology. Amsterdam: North Holland.

KORN, E. D., GreenblatT, C. L. \& Less, A. M. (1965). Synthesis of unsaturated fatty acids in the slime mold Physarum polycephalum and the zooflagellates Leishmania farentolae, Trypanosoma lewisi and Crithidia sp. A comparative study. Journal of Lipid Research 6 , 43-47.
Mangold, H. K. \& Malins, D. C. (1960). Fractionation of fats, oils and waxes on thin layers of silicic acid. Journal of the American Oil Chemists Society 37, 383-385.

Nichols, B. W. (1964). Separation of plant phospholipids and glycolipids. In New Biochemical Separations, pp. 221-237. Edited by A. T. James \& I. J. Morris. London: Van Nostrand.

PaCe-Asciak, C. \& Wolfe, L. S. (1971). A novel prostaglandin derivative formed from arachidonic acid by rat stomach homogenates. Biochemistry 10 , 3657-3664.

PITT, J. I. (1979). The Genus Penicillium and Its Teleomorphic States Eupenicillium and Talaromyces. North Ryde, Australia: Academic Press.

RadWaN, S. S. (1978). Coupling of two-dimensional thin-layer chromatography with gas chromatography for the quantitative analysis of lipid classes and their constituent fatty acids. Journal of Chromatographic Science 16, 538-542.

RadWAN, S. S. \& MaNGold, H. K. (1980). Biochemistry of lipids in plant cell cultures. In Advances in Biochemical Engineering, pp. 109-133. Edited by A. Fiechter, T. K. Ghose \& N. Blakebrough. Berlin: Springer.

RAPER, K. B. \& FenNell, D. T. (1977). Aspergillus. New York: Robert E. Krieger Publishing Company.

Siakotos, A. N. \& Rouser, G. (1965). Analytical separation of non-lipid water soluble substances and gangliosides from other lipids by dextran gel column chromatography. Journal of the American Oil Chemists Society 42, 913-919.

STAHL, E. (1962). Dünschicht-Chromatographie. Berlin: Springer. 